\title{
Congenital sucrase-isomaltase deficiency arising from cleavage and secretion of a mutant form of the enzyme
}

\author{
Ralf Jacob, ${ }^{1}$ Klaus-Peter Zimmer, ${ }^{2}$ Jacques Schmitz, ${ }^{3}$ and Hassan Y. Naim ${ }^{1}$ \\ ${ }^{1}$ Department of Physiological Chemistry, School of Veterinary Medicine Hannover, Hannover, Germany \\ ${ }^{2}$ University Children's Hospital Münster, Münster, Germany \\ ${ }^{3}$ Groupe Hospitalier, Necker-Enfants Malades, Paris, France
}

Address correspondence to: Hassan Y. Naim, Department of Physiological Chemistry, School of Veterinary Medicine, Hannover, Bünteweg 17, D-30559 Hannover, Germany. Phone: 49-511-953-8780; Fax: 49-511-953-8585;

E-mail: hnaim@biochemie.tiho-hannover.de.

Received for publication February 22, 2000, and accepted in revised form June 6, 2000.

\begin{abstract}
Congenital sucrase-isomaltase deficiency (CSID) is an autosomal recessive human intestinal disorder that is clinically characterized by fermentative diarrhea, abdominal pain, and cramps upon ingestion of sugar. The symptoms are the consequence of absent or drastically reduced enzymatic activities of sucrase and isomaltase, the components of the intestinal integral membrane glycoprotein sucrase-isomaltase (SI). Several known phenotypes of CSID result from an altered posttranslational processing of SI. We describe here a novel CSID phenotype, in which pro-SI undergoes an unusual intracellular cleavage that eliminates its transmembrane domain. Biosynthesis of pro-SI in intestinal explants and in cells transfected with the SI cDNA of this phenotype demonstrated a cleavage occurring within the endoplasmic reticulum due to a point mutation that converts a leucine to proline at residue 340 of isomaltase. Cleaved pro-SI is transported to and processed in the Golgi apparatus and is ultimately secreted into the exterior milieu as an active enzyme. To our knowledge this is the first report of a disorder whose pathogenesis results not from protein malfolding or mistargeting, but from the conversion of an integral membrane glycoprotein into a secreted species that is lost from the cell surface.
\end{abstract}

J. Clin. Invest. 106:281-287 (2000).

\section{Introduction}

Altered and defective intracellular transport, secretion, and sorting of proteins, as well as loss of function, are in many cases generated by mutations in the coding regions of the proteins. Such defects are often lethal during early stages of development or lead later on to severe disorders due to disturbances in function. An exception to this are mutations in intestinal brush border hydrolases that lead to mild diseases, such as congenital sucrase-isomaltase deficiency (CSID). CSID is an autosomal recessive intestinal disease that is characterized by the absence of the sucrase and most of the maltase digestive activity within the sucrase-isomaltase (SI) enzyme complex, with the isomaltase activity varying from absent to normal (1). Clinically, the disease is manifested as an osmotic-fermentative diarrhea upon ingestion of disaccharides and oligosaccharides. Analysis of this disorder at the molecular and subcellular levels has unraveled a number of phenotypes of CSID, which are characterized by perturbations in the intracellular transport, polarized sorting, aberrant processing, and defective function of SI (2-5). SI is a major heterodimeric membrane-bound glycoprotein of the intestinal brush border, comprising two strongly homologous subunits, sucrase and isomaltase (6). These two domains originate from a large polypeptide precursor, pro-SI, by tryptic cleavage occurring in the intestinal lumen, and they ultimately maintain a strong association through noncova- lent ionic interactions $(7,8)$. SI is a type II integral membrane glycoprotein that is synthesized with an uncleavable signal sequence, which functions also as a membrane-anchoring domain (6). This enzyme complex is a heavily N-and O-glycosylated protein (8). The earliest detectable form of pro-SI in the endoplasmic reticulum (ER) is a $210-\mathrm{kDa}$ mannose-rich form that is transported at a relatively slow rate to the Golgi apparatus, where it is processed to a complex glycosylated protein (about $245 \mathrm{kDa}$ ). O-glycosylation of pro-SI occurs mainly in a Ser/Thr-rich stalk domain located close to the membrane in the isomaltase subunit (9). This type of glycosylation has been recently shown to constitute the most critical signal for targeting of pro-SI to the apical membrane through direct association in lipid microdomains (10). The abnormal posttranslational processing of an otherwise normally synthesized pro-SI in CSID is compatible with the presence of mutations or deletions in the coding region of the SI gene. Cloning and sequencing of a cDNA of SI from an intestinal biopsy specimen of a patient with phenotype II of CSID have resulted in the identification of a point mutation that leads to a glutamine $\rightarrow$ proline substitution at residue 1098 of the sucrase subunit (Q1098P) (11). This mutation leads to a transport arrest of pro-SI in the cis-Golgi as well as in the ER and the ER-Golgi-intermediate compartment (ERGIC). To our knowledge no other molecular analyses of a CSID phenotype have been described until now. 
In this paper we describe a novel phenotype of CSID and the corresponding mutation, which results in a cleavage of membrane-bound pro-SI to a secretory protein $\left(\right.$ pro-SI $\mathrm{sec}_{\text {c }}$ ) in the ER. Pro-SI $\mathrm{sec}_{\text {in }}$ is then transported efficiently to the Golgi apparatus and secreted into the extracellular milieu as an active protein. The loss of anchored pro-SI from the membrane is therefore the basis for CSID in this phenotype.

\section{Methods}

Processing of biopsy samples. CSID in a two-year-old patient with acidic diarrhea was suggested by a sucrose tolerance test while the patient was under total parenteral nutrition. Control intestinal tissue was taken from a patient screened for diagnostic purposes other than CSID. Three biopsy samples were obtained from each patient and immediately processed as follows. One sample was cut into small pieces and fixed for immunoelectron microscopy. One sample was frozen in liquid nitrogen for enzyme activity measurements and RNA preparation. One sample was biosynthetically labeled in organ tissue culture dishes as described by Naim et al. (12).

Isolation and mutagenesis of SI CDNA. Cloning of the SI cDNA from the patient's mucosal cells followed the strategy described previously (11). Messenger RNA was isolated using Dynabeads Oligo(dT) (Dynal, Oslo, Norway) and cDNA was synthesized with the First Strand cDNA Synthesis Kit (Amersham Pharmacia Biotech, Uppsala, Sweden) using random hexamer nucleotide primers. For each PCR reaction, 1/10 $(2 \mu \mathrm{L})$ of the reaction mixture was used as a template. PCR reactions were performed with the seven primer pairs published by Ouwendijk et al. (11). The negative controls underwent the same procedure, except that cDNA template was excluded from each reaction mixture. The products of two independent PCR reactions were directly cloned into the pCR 2.1 vector (Invitrogen, Groningen, The Netherlands) and sequenced in both orientations with M13 universe and reverse primers. The sequence analysis revealed a single mutation converting $\mathrm{T}$ to $\mathrm{C}$ at position 1021 in the product encompassing nucleotides 652-1366. For transfection in Madin-Darby canine kidney (MDCK) cells, the complete SI cDNA in the plasmid vector pSG8 (phSI) (13) was mutated at position 1021 by oligonucleotide-directed mutagenesis using the Quick Change in vitro Mutagenesis System (Stratagene, La Jolla, California, USA). The following oligonucleotides were used in this context: $\mathrm{SI}_{\mathrm{T} / \mathrm{C} \text { cupstream: } 5^{\prime} \text {-TAT }}$

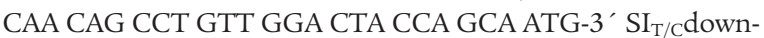
stream: 5 '-CAT TGC TGG TAG TCC AAC AGG CTG TTG ATA-3'. Mutation of $T$ to $C$ was confirmed by sequencing, and the plasmid obtained was denoted $\mathrm{pSG} 8-\mathrm{SI}_{\mathrm{T} / \mathrm{C}}$.

Transfection and metabolic labeling of MDCK cells. MDCK cells were transfected using a calcium phosphate procedure described previously (14). Forty-eight hours after transfection, MDCK cells were biosynthetically labeled with $\left.80 \mu \mathrm{Ci} \mathrm{L-[}{ }^{35} \mathrm{~S}\right]$ Redivue PRO-MIX (Amersham Pharmacia Biotech) as described by Naim et al. (15). Cell lysis was performed for 1 hour at $4^{\circ} \mathrm{C}$ in lysis buffer (25 mM Tris-HCl, pH 8.0, $50 \mathrm{mM} \mathrm{NaCl,} \mathrm{0.5 \%}$ Triton X-100, $0.5 \%$ sodium deoxycholate and a mixture of protease inhibitors containing $1 \mu \mathrm{g} / \mathrm{mL}$ pepstatin, 5 $\mu \mathrm{g} / \mathrm{mL}$ leupeptin, $17.4 \mu \mathrm{g} / \mathrm{mL}$ benzamidine, and 1 $\mu \mathrm{g} / \mathrm{mL}$ aprotinin). Usually, $1 \mathrm{~mL}$ ice-cold lysis buffer was used for each 100-mm culture dish (about $2 \times 10^{6}$ to $4 \times 10^{6}$ cells).

Immunoprecipitation. Homogenates of biosynthetically labeled biopsy specimens or cell lysates were centrifuged for 1 hour at $100,000 \mathrm{~g}$ at $4^{\circ} \mathrm{C}$, and the supernatants were immunoprecipitated as described by Naim et al. (8). Four epitope-specific mAb's directed against sucrase, isomaltase, or pro-SI were used that precipitate the high mannose glycosylated (pro-SI $\mathrm{h}_{\mathrm{h}}$ ) and the com-

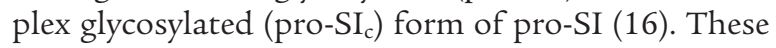
products of the hybridomas HBB 1/691, HBB 2/614, $\mathrm{HBB} 2 / 219$, and HBB 3/705 were generously provided by H.P. Hauri (Biozentrum, Basel, Switzerland) and E.E. Sterchi (Institute of Biochemistry and Molecular Biology, University of Bern, Bern, Switzerland).

Enzyme activities. Isomaltase and sucrase activities were measured according to Dahlqvist (17), using maltose and sucrose as substrates. SI was immunoprecipitated from biopsy homogenates, detergent extracts (pro-SI $\mathrm{wt}_{\mathrm{wt}}$ ), or media (pro-SI $\mathrm{sec}_{\text {) }}$ of transfected MDCK cells and was assayed for disaccharidase activity essentially as described by Naim et al. (15).

Immunoelectron microscopy. Biopsy samples were frozen in liquid nitrogen after fixation in $5 \%$ paraformaldehyde in $50 \mathrm{mM}$ HEPES and cryoprotection by polyvinylpyrrolidone and sucrose. Sectioning and labeling of ultrathin frozen sections were performed as previously described (18). Small tissue specimens were sectioned with a Leica EM ULTRACUT RFCS cryoultramicrotome (Leica, Bensheim, Germany) at $-100^{\circ} \mathrm{C}$ to $-110^{\circ} \mathrm{C}$. Thawed sections were incubated for 45 minutes with a polyclonal antiSI antibody (19) and finally with goat anti-rabbit immunogold (Dianova, Hamburg, Germany) at room temperature. The grids were contrasted with uranyl acetate, embedded in $2 \%$ methylcellulose $(1 \mathrm{~mL}$ methylcellulose contained $0.1 \mathrm{~mL} \mathrm{3 \%} \mathrm{uranyl} \mathrm{acetate),}$ and examined in a Phillips 400 electron microscope (Phillips, Eindhoven, The Netherlands).

Other procedures. Digestion of ${ }^{35} \mathrm{~S}$-labeled immunoprecipitates with endo- $\beta$ - $N$-acetylglucosaminidase $\mathrm{H}$ (Endo $\mathrm{H}$ ) and endo- $\beta$ - $N$-acetylglucosaminidase $\mathrm{F} / \mathrm{gly}$ copeptidase $\mathrm{F}$ (Endo $\mathrm{F} / \mathrm{GF}$ ) was performed as previously described (15). Triton X-114 (TX-114) extraction was performed essentially as described by Wilson et al. (20). SDS-PAGE was performed according to Laemmli (21). As molecular weight references, high molecular weight markers from Sigma Aldrich (Deisenhofen, Germany) were used.

\section{Results}

Assessment of CSID. A sucrose tolerance test initially suggested that the patient had sucrose malabsorption. This was confirmed by measurement of sucrase and 
isomaltase enzymatic activities in the homogenates of a biopsy specimen. The control employed another disaccharidase, lactase-phlorizin hydrolase (LPH). While the activity of LPH was in the normal range (16-32 $\mathrm{IU} / \mathrm{mg}$ protein), the activities of sucrase and isomaltase were below detection levels, pointing to an enzymatic defect restricted to SI.

Biosynthesis, processing, and subcellular localization of SI in the patient's tissue. To determine whether this deficiency is the result of an altered posttranslational processing of or a block in the transport of pro-SI along the secretory pathway, we analyzed the structural features of pro-SI in a biosynthetically labeled patient's biopsy specimen. A schematic drawing of the structure and membrane association of pro-SI is shown in Figure 1. Immunoprecipitation of SI from detergent extracts of the labeled tissue revealed a protein band of an apparent molecular weight of $210 \mathrm{kDa}$. This band had a slightly diffuse pattern when directly compared with its counterpart in the control biopsy (Figure 2a). This observation suggested the presence of different glycosylated forms of the same polypeptide or, alternatively, polypeptides with different sizes. Treatment with Endo H, which cleaves mannose-rich glycans, resolved the patient's pro-SI into two definite polypeptides with slightly different apparent weights. Control pro-SI, on the other hand, shifted to one single polypeptide $(185 \mathrm{kDa})$ under similar treatment conditions. Comparison of the Endo $\mathrm{H}$ digests revealed that the upper band of the doublet in the patient's pro-SI had a similar apparent molecular weight to that of the $185-\mathrm{kDa}$ product of the control species (Figure $2 \mathrm{~b}$ ). The Endo H sensitivity of the $210-\mathrm{kDa}$ polypeptide is compatible with a mannose-rich type of glycosylation of pro-SI, as well as with an ER localization. Since two polypeptides in the patient's tissue, compared with one in the control, were generated upon N-deglycosy- lation, we concluded that the smaller Endo $\mathrm{H}$ band corresponds to a truncated form of the full-length mannose-rich pro-SI species. After 4 hours of pulse labeling, no change in the electrophoretic band pattern in the patient's pro-SI was observed as compared with the previous time point. Likewise, the Endo $\mathrm{H}$ sensitivity pattern of this protein band was maintained. By contrast, the control biopsy specimen revealed, at a similar labeling time point, a $245-\mathrm{kDa}$ complex glycosylated and mature Endo H-resistant form (Figure $2 \mathrm{~b}$ ) reminiscent of processing and maturation in the Golgi apparatus prior to targeting to the apical membrane (8). It is obvious, therefore, that abnormalities in the posttranslational processing of an otherwise normally synthesized pro-SI protein have occurred in the patient's specimen. To examine whether these alterations were restricted to pro-SI or were due to a general cellular defect, the biosynthesis of other control brush border enzymes was investigated. LPH and aminopeptidase N (ApN) were synthesized and processed into complex glycosylated species in the patient's intestinal cells, similarly to control cells, thus ruling out a general cellular defect as the underlying cause of the abnormal biosynthetic pathway of pro-SI (Figure 2c) (12).

At this stage of investigation, it was not clear whether the exclusive detection of pro-SI as an Endo H-sensitive protein in the patient's biopsy sample, despite 4 hours of labeling, was an indication of an ER block, or of a probably different and unknown processing mechanism that remained to be delineated.

We analyzed the subcellular localization of pro-SI in the patient's epithelial cells by immunogold labeling. Figure 3 shows a slightly stronger labeling of the ER in the patient's tissue (inset in c) than in the control biopsy specimen; the labeling of the Golgi cisternae was comparable in both samples. The control tissue, on the other hand, a

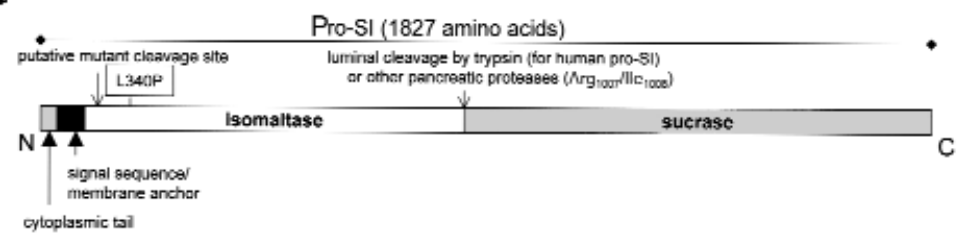

b

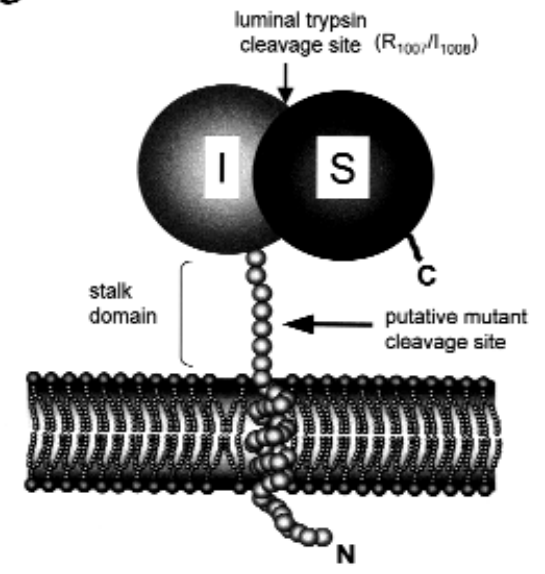

Figure 1

Structure and membrane orientation of pro-SI. (a) Structural features of pro$\mathrm{SI}$ deduced from biosynthetic studies (8) and cDNA cloning (6). Pro-SI is a type II membrane glycoprotein $\left(\mathrm{N}_{\text {in }} / \mathrm{C}_{\text {out }}\right)$ that is synthesized with an uncleavable signal sequence, which also serves as a membrane anchoring domain (6). The cytoplasmic tail contains 12 amino acid residues and is followed by a membrane anchor of 20 amino acids and a Ser/Thr-rich stalk domain of 28 amino acids that is considered to be part of the isomaltase subunit. Isomaltase ends with amino acid residue Arg $_{1007}$ and sucrase starts immediately thereafter with $\mathrm{ll}_{1008}$. The location of the L340P mutation in the CSID patient and the putative cleavage site are indicated. The Arg/lle peptide sequence between isomaltase and sucrase is a trypsin site where the mature large precursor pro-SI is cleaved in the intestinal lumen by pancreatic trypsin (16). (b) Schematic drawing of the membrane orientation of pro-SI. The $\mathrm{NH}_{2}$-terminus on the cytosolic side of the membrane $(\mathrm{N})$, the luminal $\mathrm{COOH}$-terminus $(\mathrm{C})$, the stalk domain, and the putative cleavage region are indicated. I, isomaltase; $\mathrm{S}$, sucrase. 
a

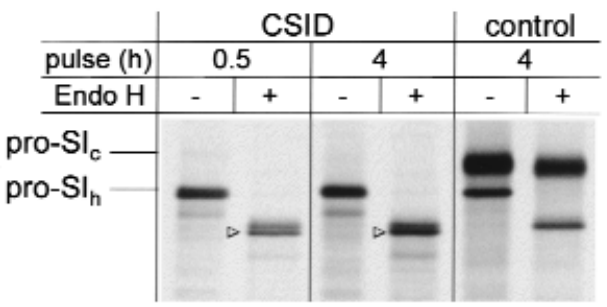

b

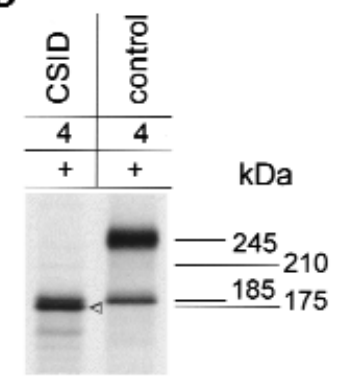

C

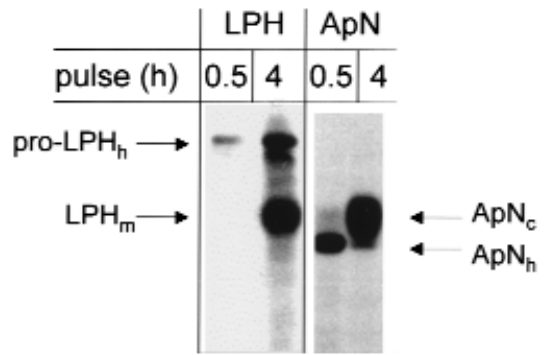

\section{Figure 2}

Molecular forms of SI in CSID and in normal control. Biopsy samples from a patient with CSID and a normal control were biosynthetically labeled for the indicated times with ${ }^{35} \mathrm{~S}$-methionine. (a) The specimens were homogenized, solubilized, and immunoprecipitated with monoclonal anti-SI antibodies. The immunoprecipitates were divided into two equal parts; one part was treated with Endo $\mathrm{H}$ and the other was not treated. Finally the samples were subjected to SDS-PAGE on $6 \%$ slab gels. Gels were analyzed by fluorography. (b) Direct comparison of Endo $\mathrm{H}$-treated immunoprecipitates from the patient with CSID and the control shown in a. The additional band appearing in the CSID samples in $\mathbf{a}$ and $\mathbf{b}$ is indicated by an arrowhead. (c) The detergent extracts of the biopsy samples from the patient and the control were also immunoprecipitated with mAb's directed against LPH and aminopeptidase $N(A p N)$, which served as control brush border glycoproteins. The immunoprecipitates were analyzed on SDS-PAGE on $6 \%$ slab gels.

revealed intense labeling, confined to the brush border membrane (5), that could not be detected in the patient's tissue. Both the patient and the control samples did not show any labeling of the basolateral membrane. The total number of gold particles was several orders of magnitude higher in the control biopsy than in the patient's tissue and did not correlate therefore with the normal synthetic levels deduced from the biochemical data. One explanation for these differences between the morphological and biochemical data was that the truncated form of proSI lacked the membrane anchoring domain, was a hydrophilic and transport-competent protein that acquired complex glycans in the Golgi apparatus and was secreted into the external milieu. In this case the complex glycosylated form barely would be detected in the cell lysates at the protein level, as had been recently demonstrated for a soluble mutant of the pro-SI molecule (10). In fact, a substantial proportion of the protein was not present in the cell at steady state, which was clearly demonstrated by the faint immunogold labeling. A possible interpretation based only on the biochemical data was that pro-SI and its truncated form in the patient's tissue did not acquire a complex type of glycans in the Golgi apparatus and had been blocked intracellularly in the ER or in a pre-Golgi compartment. This is only partially supported by the morphological data, since a difference between the labeling intensities of the ERs in the two samples was detected.

Isolation of an SI cDNA from the patient's tissue and identification of a point mutation responsible for the CSID phenotype. The normal synthetic levels of SI in the patient's biopsy sample and the altered posttranslational behavior strongly supported the notion that a mutation or a deletion in the coding region of the SI gene was responsible for the generation of this SI phenotype. An isolation of the SI cDNA, therefore, would allow the identification of changes in the cDNA possibly responsible for this phenotype. Furthermore, expression of the cDNA in a heterologous transfection system would help to identify the processing and trafficking steps of pro-SI that were impossible to perform due to tissue limitation; thus it would delineate essential details of the molecular basis of this novel phenotype.

To isolate the SI cDNA we followed a strategy essentially similar to that used in the first identification of a mutation in a phenotype of CSID (11). The products of two independent PCR reactions were cloned into $\mathrm{PCR}$ 2.1 vector and were sequenced from the flanking M13 universe and reverse primer sites in the vector. The sequence analysis revealed one alteration relative to the wild-type sequence (22). This alteration, T/C at nucleotide 1021, was found in the sequence encoding the isomaltase subunit of the pro-SI complex and resulted in a leucine $\rightarrow$ proline substitution at amino acid residue 340 (L340P). Sequencing of several independent PCR products confirmed the presence of this single point mutation in the patient's cDNA. The wildtype sequence never could be identified, suggesting that both alleles of the SI gene contained this mutation or, more likely, that the wild-type allele was not expressed.

Transient expression of the mutant SI CDNA in mammalian cells and characterization of the biosynthesis, processing, folding, and function of mutant pro-SI. We wanted next to determine whether the L340P substitution was the underlying cause of the altered posttranslational processing of pro-SI in the new phenotype. For this purpose we introduced this mutation into the wild-type SI cDNA by using oligonucleotide-directed mutagenesis. The resulting mutant was then analyzed in transiently transfected MDCK cells. Figure 4a shows immunoprecipitated mutant pro-SI from transfected cells that were subjected to a pulse-chase labeling protocol. At early chase points, pro-SI isolated from the cell lysates had a band pattern similar to that discerned in the detergent 
extracts of the biopsy sample. Pro-SI was Endo H-sensitive and shifted to a double band of slightly different apparent molecular weights. At 1 hour of chase, the mannose-rich pro-SI still was detected and was resolved, as in the previous time point, into a doublet. Additionally, a predominantly Endo $\mathrm{H}$-resistant band appeared, indicating that this form had acquired complex glycosylated glycans in the Golgi apparatus. This Endo $\mathrm{H}$-resistant band was secreted into the culture, and its labeling intensity was severalfold higher than in the lysates. Three hours into the chase, the mannose-rich pro-SI species no longer was resolved into a doublet upon Endo H treatment and only the lower band was detected. This indicated that the truncated pro-SI was the predominant form, and its complex glycosylated Endo H-resistant form was found mainly in the culture medium and barely was visible in the cell lysates. At 6 hours of chase, a pattern similar to that at 3 hours of chase was obtained. The pulse-chase data demonstrated, therefore, that the truncated form traversed the Golgi apparatus where it acquired complex glycans and ultimately was secreted into the exterior milieu. A similar secreted form could not be detected in the control cells that were transfected with wild-type SI cDNA, which was anticipated, since SI is an integral membrane protein. The secreted pro-SI mutant was predominantly Endo H-resistant, clearly indicating that it had traversed the Golgi apparatus, where the majority of the $\mathrm{N}$ glycans had acquired complex type of glycosylation.

It is apparent in light of the pulse-chase data that the truncated form of pro-SI was a precursor of the secreted species and as such was also a hydrophilic protein. To confirm this we examined the detergent extractability in TX-114 of the pro-SI forms from the patient and control tissues. Figure $4 \mathrm{~b}$ demonstrates that the truncated mannose-rich pro-SI form was recovered in the soluble phase whereas the full-length pro-SI was found predominantly in the detergent phase. Likewise, the mannose-rich pro-SI derived from the control sample was found exclusively in the detergent phase. Altogether, the data support the notion that the mannoserich truncated pro-SI form was as hydrophilic as its secreted complex glycosylated counterpart. To determine the site of intracellular cleavage, the pulse labeling was performed with transfected cells at $15^{\circ} \mathrm{C}$. At this temperature constitutive transport of proteins is blocked in the ER and possibly also the ERGIC. Figure $4 \mathrm{~b}$ shows that the mutant pro-SI doublet was already present at $15^{\circ} \mathrm{C}$, indicating that cleavage had already occurred in the ER or ERGIC. The pulse-chase data have clearly indicated that the truncated form of proSI was a transport-competent molecule.

Finally, to determine whether the secreted form was enzymatically active, we compared its activity with that of wild-type pro-SI. The secreted pro-SI mutant was isolated from the culture media, while wild-type pro-SI was isolated from detergent extracts of transfected cells. The sucrase activity in the secreted form was 31.2 IU, similar to that in wild-type SI (35.6 IU). The isoma- ltase activity was also comparable with its counterpart in the wild type-species (28.4 compared with $25.9 \mathrm{IU})$. It should be noted that the cell homogenates did not contain detectable sucrase or isomaltase activities. Since the secreted form expressed normal sucrase as well as isomaltase enzymatic activities, we concluded that the intracellular cleavage of mutant pro-SI took place in a region downstream of the catalytic sites of sucrase and isomaltase, which are located at amino acid residues 1540 and 503, respectively. Also, this is obvious when one considers the size of the secreted form which was compatible with the cleavage of approximately 40 amino acids from the full-length pro-SI.

Altogether, the amino acid substitution L340P has resulted in the generation of a secreted form of pro-SI. The presence of two mannose-rich species and the

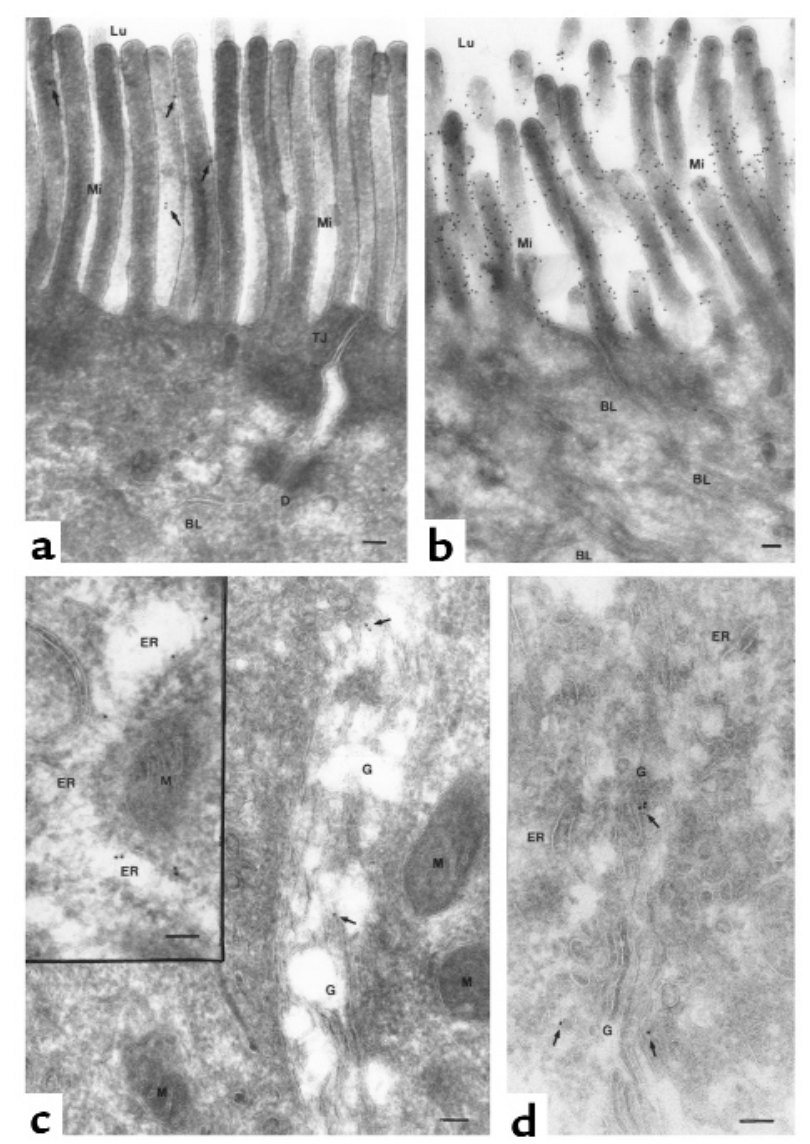

Figure 3

Ultrastructural localization of SI in biopsy specimens of CSID and a normal control. SI was localized on thin frozen sections of small bowel biopsies of the patient ( $\mathbf{a}$ and $\mathbf{c}$ ) and the control (b and $\mathbf{d}$ ), which were labeled by a polyclonal antibody against SI and by 12-nm large goat anti-rabbit immunogold particles. The apical membranes (arrows) of affected enterocytes show only a weak labeling by the SI antibody, in contrast to control enterocytes. The basolateral membranes of enterocytes are free of labeling. While there is no difference in the labeling densities within Golgi stacks between patient and control enterocytes, the ER contains a slightly increased number of gold particles in affected enterocytes compared with control cells. Mi, microvilli; Lu, lumen; TJ, tight junction; BL, basolateral membrane; D, desmosome; G, Golgi apparatus; M, mitochondria. Scale bars: $0.1 \mu \mathrm{m}$. 
a

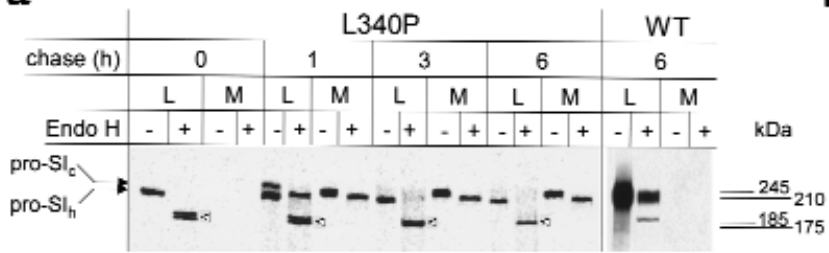

b

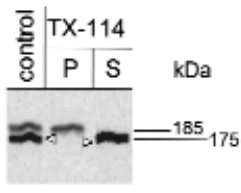

\section{Figure 4}

Expression of mutant SI cDNA in MDCK cells. (a) MDCK cells were transfected with phSI (wild-type [WT]) and pSG8-SIT/C (L340P) and biosynthetically labeled with ${ }^{35} \mathrm{~S}$-methionine for 1 hour followed by a chase period for the indicated times. The cell lysates $(\mathrm{L})$ and the culture media (M) were separately immunoprecipitated with mAb anti-SI. Thereafter the immunoprecipitates were divided into equal parts and treated or not treated with Endo $\mathrm{H}$; they were analyzed by SDS-PAGE on 6\% slab gels and by fluorography. (b) MDCK cells were transfected with pSG8$\mathrm{SI}_{\mathrm{T} / \mathrm{C}}$, and 48 hours after transfection they were labeled at $15^{\circ} \mathrm{C}$ with ${ }^{35} \mathrm{~S}$-methionine for 6 hours. Lysis was performed in the presence of TX114. SI was immunoprecipitated from the aqueous $(\mathrm{S})$ and the detergent $(\mathrm{P})$ phase, treated with Endo $\mathrm{H}$, and analyzed by SDS-PAGE on $6 \%$ slab gels followed by fluorography. The additional band appearing in the L340P samples in $\mathbf{a}$ and $\mathbf{b}$ is indicated by arrowheads.

product-product relationship between these forms clearly indicated that the secreted molecule was derived from membrane-bound pro-SI by intracellular processing in the ER or in a pre-Golgi compartment.

\section{Discussion}

A common feature of known phenotypes of SI in CSID is the synthesis of normal levels of an enzymatically inactive protein that is blocked intracellularly or mistargeted to the basolateral membrane. In all these cases SI is neither accessible to nor able to hydrolyze its substrates (2-5). Initially it had been proposed that the molecular bases of these phenotypes are mutations in the coding region of the SI gene $(4,5)$. An experimental support to this hypothesis was provided by the first identification of a mutation in the CDNA of SI in one of the CSID phenotypes, namely phenotype II. In this phenotype, pro-SI is blocked intracellularly in the ER, ERGIC, and cis-Golgi due to a structural alteration in the sucrase subunit generated by a mutation that converts a glutamine to a proline at amino acid residue 1098 (11). The characterized mutation may have generated a retention signal in sucrase that is implicated in a quality control mechanism that operates beyond the ER and prevents the mutant molecule from being transported further to its site of action. The current investigation describes the molecular and subcellular characterization of a novel phenotype of SI in CSID. This phenotype does not share the common features of the previously investigated phenotpes, and yet it causes CSID. The most striking and novel feature of this phenotype is that an enzymatically active and transport-competent form of pro-SI is expressed, which, however, does not exert its function normally in the intestine. In this phenotype, pro-SI undergoes an early intracellular cleavage that leads to the generation of a soluble mannose-rich form that is transport-competent, transpasses the Golgi apparatus, and is secreted out of the cell as an enzymatically active molecule. However, it appears that the secreted form is not adequately exposed to its substrate in the intestinal lumen because CSID has been assessed using the hydrogen breath test. This is possibly the result of a short half-life of the secreted form.
The generation of the secreted hydrophilic form of SI occurs through the elimination of its membrane anchoring domain, which also serves as a signal sequence in this type II membrane glycoprotein. We were able to characterize the molecular basis of this cleavage, which is a point mutation at nucleotide 1021 that results in a leucine $\rightarrow$ proline substitution at amino acid residue 340 of the isomaltase subunit. This mutation is indeed responsible for this phenotype, since introduction of the L340P into wild-type pro-SI in a transfected mammalian cell line generates effects similar to those observed in the intestinal tissue. How could this mutation generate a cleaved form of pro-SI?

It is known that the primary sequence and the conformation of the region around the membrane anchor/signal sequence of type II integral membrane proteins have a decisive influence on the cleavage by signal peptidase (23-27). The deletion of the cytoplasmic tail or the insertion of a hydrophilic segment into the transmembrane domain of neutral endopeptidase, for instance, results in increased processing by signal peptidase (23). For pro-SI the uncleavable membrane anchor/signal sequence can be converted into a processed form by the substitution of a single proline in the transmembrane domain (28), emphasizing the susceptibility of pro-SI for signal peptidase processing. Additionally, sequences upstream of the cleavage site have profound influences on signal peptide function and signal peptidase cleavage, as has been shown for prolactin (24). Although the mutation lies in a region 308 amino acids apart from the membrane anchor, it is unlikely that the cleavage has taken place at the mutation. In fact, the molecular weight difference of no more than $10-\mathrm{kDa}$ between the membrane-bound and secreted form of pro-SI is too small to be compatible with a cleavage of more than 300 amino acids. It is likely that the mutation has induced subtle alteration in the region near the membrane anchor, rendering it accessible to a signal peptidase. One of the known characteristics of proline within secondary structures of proteins is its high alpha-helix-breaking potential (29). One would therefore expect a leucine $\rightarrow$ proline substitution at position 340 to be associated with dramatic effects on 
the three-dimensional structure of a small protein subdomain next to the membrane anchor, rendering it susceptible to cleavage by a protease. The signal peptidase is likely to be responsible for the generation of the secreted pro-SI ${ }_{\mathrm{sec}}$ due to the early stage of the cleavage process. This is strongly suggested by the mannose-rich type of glycosylation of the cleaved form.

Perhaps the most significant finding of this investigation is that a disease occurs despite the expression of an active and transport-competent protein because this protein is secreted and therefore does not reside in its site of action. The pathogenesis of many well investigated disorders is often associated with altered processing and impaired transport of cell surface proteins. In most of these cases the protein is blocked intracellularly, often in the ER, and is ultimately degraded. Examples of this phenotype are the $\Delta \mathrm{F} 508$ mutant of the cystic fibrosis transmembrane conductance regulator (30), several cases of the sodium-glucose transporter in glucose galactose malabsorption (31), and mutants of the LDL receptor in familial hypercholesterinemia (32). Phenotype I of SI in CSID also undergoes a transport block in the ER $(4,5)$. To our knowledge, a molecular mechanism similar to this novel CSID phenotype that is responsible for the pathogenesis of a genetic disorder has not been reported before for a cell surface protein. In this respect, the CSID phenotype presented here is novel not only for this particular genetic disorder but even for other inherited diseases.

\section{Acknowledgments}

We are grateful to Jürgen Eikemeyer and Dagmar Bühring for excellent technical assistance. We thank Hans-Peter Hauri and Erwin Sterchi for generous gifts of the monoclonal anti-SI antibody, and Alain Zweibaum (Unité de Recherches sur la Différenciation Cellulaire Intestinale, Institut National de la Santé et de la Recherche Médicale, Villejuif, France) for his generous gift of the polyclonal anti-SI antibody. We thank Catherine Lenaerts (University Hospital Amiens, Amiens, France) for providing the access to her patient. This work was supported by grants $\mathrm{Na}$ 331/1-2 from the Deutsche Forschungsgemeinschaft and A16 from the Sonderforschungsbereich 280 (Bonn, Germany; both to H.Y. Naim).

1. Kerry, K.R., and Townley, R.R.W. 1965. Genetic aspects of intestinal sucraseisomaltase deficiency. Aust. Paediatr. J. 1:223-235.

2. Hauri, H.P., Roth, J., Sterchi, E.E., and Lentze, M.J. 1985. Transport to cell surface of intestinal sucrase-isomaltase is blocked in the Golgi apparatus in a patient with congenital sucrase-isomaltase deficiency. Proc. Natl. Acad. Sci. USA. 82:4423-4427.

3. Lloyd, M.L., and Olsen, W.A. 1987. A study of the molecular pathology of sucrase-isomaltase deficiency. A defect in the intracellular processing of the enzyme. N. Engl.J. Med. 316:438-442.

4. Naim, H.Y., et al. 1988. Sucrase-isomaltase deficiency in humans. Different mutations disrupt intracellular transport, processing, and function of an intestinal brush border enzyme. J. Clin. Invest. 82:667-679.

5. Fransen, J.A., Hauri, H.P., Ginsel, L.A., and Naim, H.Y. 1991. Naturally occurring mutations in intestinal sucrase-isomaltase provide evidence for the existence of an intracellular sorting signal in the isomaltase subunit. $J$. Cell Biol. 115:45-57.

6. Hunziker, W., Spiess, M., Semenza, G., and Lodish, H.F. 1986. The sucraseisomaltase complex: primary structure, membrane-orientation, and evolution of a stalked, intrinsic brush border protein. Cell. 46:227-234.
7. Hauri, H.P., Quaroni, A., and Isselbacher, K.J. 1979. Biogenesis of intestinal plasma membrane: posttranslational route and cleavage of sucrase-isomaltase. Proc. Natl. Acad. Sci. USA. 76:5183-5186.

8. Naim, H.Y., Sterchi, E.E., and Lentze, M.J. 1988. Biosynthesis of the human sucrase-isomaltase complex. Differential O-glycosylation of the sucrase subunit correlates with its position within the enzyme complex. J. Biol. Chem. 263:7242-7253.

9. Jacob, R., Alfalah, M., Grunberg, J., Obendorf, M., and Naim, H.Y. 2000. Structural determinants required for apical sorting of an intestinal brushborder membrane protein. J. Biol. Chem. 275:6566-6572.

10. Alfalah, M., et al. 1999. O-linked glycans mediate apical sorting of human intestinal sucrase-isomaltase through association with lipid rafts. Curr. Biol. 9:593-596.

11. Ouwendijk, J., et al. 1996. Congenital sucrase-isomaltase deficiency. Identification of a glutamine to proline substitution that leads to a transport block of sucrase-isomaltase in a pre-Golgi compartment. J. Clin. Invest. 97:633-641.

12. Naim, H.Y., Sterchi, E.E., and Lentze, M.J. 1987. Biosynthesis and maturation of lactase-phlorizin hydrolase in the human small intestinal epithelial cells. Biochem. J. 241:427-434.

13. Moolenaar, C.E., et al. 1997. A mutation in a highly conserved region in brush-border sucrase-isomaltase and lysosomal alpha-glucosidase results in Golgi retention. J. Cell Sci. 110:557-567.

14. Jacob, R, et al. 1999. Hierarchy of sorting signals in chimeras of intestinal lactase-phlorizin hydrolase and the influenza virus hemagglutinin. J. Biol. Chem. 274:8061-8067.

15. Naim, H.Y., Lacey, S.W., Sambrook, J.F., and Gething, M.J. 1991. Expression of a full-length cDNA coding for human intestinal lactase-phlorizin hydrolase reveals an uncleaved, enzymatically active, and transport-competent protein. J. Biol. Chem. 266:12313-12320.

16. Hauri, H.P., Sterchi, E.E., Bienz, D., Fransen, J.A., and Marxer, A. 1985. Expression and intracellular transport of microvillus membrane hydrolases in human intestinal epithelial cells. J. Cell Biol. 101:838-851.

17. Dahlqvist, A. 1968. Assay of intestinal disaccharidases. Anal. Biochem. 22:99-107.

18. Zimmer, K.P., et al. 1995. Ultrastructural, immunocytochemical and stereological investigation of hepatocytes in a patient with the mutation of the ornithine transcarbamylase gene. Eur. J. Cell Biol. 67:73-83.

19. Trugnan, G., Rousset, M., Chantret, I., Barbat, A., and Zweibaum, A. 1987. The posttranslational processing of sucrase-isomaltase in HT-29 cells is a function of their state of enterocytic differentiation. J. Cell Biol. 104:1199-1205.

20. Wilson, J.M., Whitney, J.A., and Neutra, M.R. 1987. Identification of an endosomal antigen specific to absorptive cells of suckling rat ileum. J. Cell Biol. 105:691-703.

21. Laemmli, U.K. 1970. Cleavage of structural proteins during the assembly of the head of bacteriophage T4. Nature. 227:680-685.

22. Chantret, I., et al. 1992. Sequence of the complete cDNA and the $5^{\prime}$ structure of the human sucrase-isomaltase gene. Possible homology with a yeast glucoamylase. Biochem. J. 285:915-923.

23. Roy, P., Chatellard, C., Lemay, G., Crine, P., and Boileau, G. 1993. Transformation of the signal peptide/membrane anchor domain of a type II transmembrane protein into a cleavable signal peptide. J. Biol. Chem. 268:2699-2704.

24. Andrews, D.W., Perara, E., Lesser, C., and Lingappa, V.R. 1988. Sequences beyond the cleavage site influence signal peptide function. J. Biol. Chem. 263:15791-15798

25. Wiren, K.M., et al. 1989. Mutations in signal sequence cleavage domain of preproparathyroid hormone alter protein translocation, signal sequence cleavage, and membrane-binding properties. Mol. Endocrinol. 3:240-250.

26. Kohara, A., Yamamoto, Y., and Kikuchi, M. 1991. Alteration of N-terminal residues of mature human lysozyme affects its secretion in yeast and translocation into canine microsomal vesicles. J. Biol. Chem. 266:20363-20368

27. Hogue, B.G., and Nayak, D.P. 1994. Deletion mutation in the signal anchor domain activates cleavage of the influenza virus neuraminidase, a type II transmembrane protein. J. Gen. Virol. 75:1015-1022.

28. Hegner, M., et al. 1992. Single amino acid substitutions can convert the uncleaved signal-anchor of sucrase-isomaltase to a cleaved signal sequence. J. Biol. Chem. 267:16928-16933.

29. Kim, M.K., and Kang, Y.K. 1999. Positional preference of proline in alphahelices. Protein Sci. 8:1492-1499.

30. Cheng, S.H., et al. 1990. Defective intracellular transport and processing of CFTR is the molecular basis of most cystic fibrosis. Cell. 63:827-834.

31. Martin, M.G., et al. 1997. Compound missense mutations in the sodium/D-glucose cotransporter result in trafficking defects. Gastroenterology. 112:1206-1212.

32. Ko, K.W., et al. 1998. Mutation at the processing site of chicken low density lipoprotein receptor-related protein impairs efficient endoplasmic reticulum exit, but proteolytic cleavage is not essential for its endocytic functions. J. Biol. Chem. 273:27779-27785 